\title{
Randomized, Double Blind, Placebo Controlled Trial of Hydrosoluble Ubiquinol and Carnitine in Patients with Heart Failure: Longterm Follow up Results in the Tishcon Study
}

\author{
Viola Mechirova ${ }^{1}$, Adarsh Kumar ${ }^{2}$, Ram B. Singh*, ${ }^{3}$, Rakesh Sharma ${ }^{4}$, Pronobesh Chattopadhyay ${ }^{3}$, \\ Mohammad A. Niaz ${ }^{3}$, Amar S. Thakur ${ }^{3}$, Jan Fedacko ${ }^{1}$, Daniel Pella ${ }^{1}$ and Lekh Juneja ${ }^{5}$ \\ ${ }^{1}$ Faculty of Medicine, Safaric University,Kosice, Slovakia; ${ }^{2}$ Government Medical College, Amritsar, India; ${ }^{3}$ Halberg \\ Hospital and Research Institute, Moradabad, India; ${ }^{4}$ Deaprtment of Medicine, Columbia University, New York, NY, \\ USA and ${ }^{5}$ Taiyo Kagaku Co. Ltd, Yokkaichi, Japan
}

\begin{abstract}
Background: Carnitine and coenzyme Q10 are important for myocardial- mitochondrial function, and are deficient in patients with congestive heart failure. It is possible that supplementation with ubiquinol + 1- carnitine may be protective among these patients, which may improve the quality of life and morbidity in heart failure.

Subjects and Methods: In this controlled trial, the effects of carni Q-gel (L-carnitine fumarate 2250mg/day+hydrosoluble reduced ubiquinol 270mg/day) were examined for 15 months, in 31 (intervention group A) and another 31(control group B) patients with heart failure. Hospitalization due to worsening of heart failure, heart transplantation, and deaths were the combined endpoints.

Results: Echo cardiographic ejection fraction was lower at baseline (38.8 \pm 7.6 v/s $39.3 \pm 6.7 \%)$ among both the group of patients, indicating class II- IV heart failure.

Baseline serum CoQ10 $(0.21 \pm 0.11 \mathrm{v} / \mathrm{s} 0.19+0.10 \mathrm{ug} / \mathrm{ml})$ was low, however, after 12 weeks, serum CoQ showed a significant increase in the carniqgel group compared to the control group $(2.7 \pm 1.2 \mathrm{v} / \mathrm{s} 0.76 \pm 0.14 \mathrm{ug} / \mathrm{ml})$. After treatment for 12 months, the quality of life visual analogous scale revealed that dyspnea, palpitation and fatigue and NYHA class II-III-IV, which were present at rest, in all the patients, at baseline, showed beneficial effects in the intervention group compared to the placebo group. Six minutes walk test showed that there was a significant greater benefit in walking in the intervention group $(215 \pm 17.6 \mathrm{v} / \mathrm{s} 281 \pm 20.6, \mathrm{P}<0.02)$ compared to the placebo group $(218.4 \pm 17.6 \mathrm{v} / \mathrm{s} 245.7 \pm 17.1, \mathrm{P}<0.05)$. A change in the symptom scale showed that majority of the patients had improvement in the intervention group compared to the control group respectively ( $28 \mathrm{v} / \mathrm{s} 12$ patients, $\mathrm{P}<0.02)$. The deaths ( $3 \mathrm{vs} 8)$ and hospitalizations due to worsening of heart failure ( 2 vs 11) among intervention and control group respectively, were significantly lower in the carniqgel group compared to the control group (5 vs $19, \mathrm{P}<0.02$ ). Five patients had nausea and vomiting, which were controlled with symptomatic treatment. Treatment with carniqgel was stopped after 12 months. Follow up after another 3 months (total 15 months) revealed that there was a worsening of NYHA class heart failure, as well as in the quality of life symptom scale and physical performance, assessed by 6-min walk test. There was a nonsignificant increase in hospitalizations in the intervention group after cessation of carniqgel softsules, compared to hospitalizations during the last 3 months.

Conclusions: The findings indicate that treatment with ubiquinol $+\mathrm{L}-$ carnitine fumarate can cause a significant improvement in the quality of life, exercise capacity, as well as NYHA heart failure, which became worst after cessation of CoQ. There was a significant greater benefit in combined endpoints (hospitalization and deaths) in the intervention group than in the control group.
\end{abstract}

Key Words: Antioxidant, quality of life, bio energetic, heart failure, free radical stress, mitochondria.

\section{INTRODUCTION}

Chronic heart failure (CHF) may be associated with substantial morbidity and mortality, despite available treatment [1-3]. It is possible that CHF worsens due to loss of CoQ from the myocardium, as well as due to its progression because of activation of neurohormones and pro-inflammatory cytokines, following an initial cardiac injury such as myocardial ischemia, statin toxicity or a mutation of the genetic

*Address correspondence to this author at the Halberg Hospital and Research Institute, Civil Lines, Moradabad-10(UP)244001, India;

E-mail: icn2008@mickyonline.com; icn2005@sancharnet.in programme [1-8]. It seems that possible targets in the treatment of CHF involve pro-and anti- inflammatory cytokines and their receptors, endotoxin, adhesion molecules, nitric oxide and nitric oxide synthase, reactive oxygen species, and deficiency of $\mathrm{CoQ}$ and L- carnitine in mitochondria and different types of leucocytes [2-10]. Several hypotheses have been suggested to describe the origin of immune activation in CHF [3].

Coenzyme Q10 $(\mathrm{CoQ})$ is a potent antioxidant and bioenergetic agent which is rich in mitochondria of cardiomyocyte $[9,10]$. Its bioenergetic property appears to be of fundamental importance, particularly in cells with high meta- 
bolic demand such as cardiomyocyte. In chronic heart failure, its deficiency may be directly proportional to the cardiorespiratory impairment. It has been proposed that an improvement in left ventricular function and contractility may be obtained by raising plasma CoQ levels [4]. However, in advanced heart failure and coronary disease, oral CoQ supplementation in doses of close to $100 \mathrm{mg} /$ day, improved the left ventricular systolic function in several studies [10-15], but a few studies [16, 17], showed no such effects. The lack of beneficial effects may be due to the use of less bioactive CoQ preparations and lower dosages. In chronic heart failure, endothelial dysfunction contributes to functional impairment, which may be modulated by oral CoQ supplementation resulting into better endothelial-dependent relaxation of coronary as well as peripheral arteries causing better functional capacity of the patients $[4,10]$. There is evidence that coenzyme $\mathrm{Q}$ and carnitine may be beneficial in patients with heart failure [4-15, 18].

In the present study, we examine, the longterm effects of ubiquinol, in conjunction with L-carnitine, on the quality of life and NYHA classes heart failure in patients with CHF.

\section{SUBJECTS AND METHODS}

A double blind, placebo controlled, randomized trial [19] was designed including patients with CHF of mixed etiology; ischemic, hypertensive, valvular heart disease and idiopathic dilated cardiomyopathy. The status of CHF was assessed by New York Heart Association (NYHA) and categorized into functional Class II to IV. The quality of life was assessed by visual analogous function rating scale and 6minute walk test. Dyspnea was graded into no dyspnea, dyspnea at moderate exercise, dyspnea at mild exercise, and dyspnea at rest on a scale and subjects having dyspnea were graded by marking on the scale in all the subjects. Fatigue was classified into never, occasionally, frequently and always on a scale and subjects having fatigue were graded by marking on the scale. Symptoms changes after administering treatment, were also marked on a scale, as deterioration, no change and improvement. Physical performance was assessed by a 6-min walk test that is distance walked in 6 minutes.

All patients participated with a written informed consent and the study was approved by the institutional ethic committee. All the patients were recruited from the Department of Cardiology, Government Medical College, Amritsar, India, for this study. Five patients were excluded due to various reasons and remaining 62 patients presenting with CHF were randomized to intervention ( $\mathrm{Gr} \mathrm{A}, \mathrm{n}=31)$ and placebo groups $(\mathrm{Gr} B, \mathrm{n}=31)$ by computer generated random numbers. The physician, laboratory technician and patients were blind to the treatment groups.

Patients with hypertrophic cardiomyopathy $(\mathrm{n}=1)$, amyloid cardiomyopathy $(n=1)$ and those not willing to give consent $(n=2)$ were excluded. Patients presenting with shock, $(n=1)$ chronic renal failure and cancer were also excluded before randomization of the patients.

We administered Carni Q-gel, 9 softgels (3 with each meal thrice daily), which provide a total of $270 \mathrm{mg}$ ubiquinol and $2250 \mathrm{mg}$ - L-carnitine daily - or 9 matching placebos daily to each patient, during the period of 12 months. All patients were kept on standard drug therapy, and carni Q-Gel and placebo were used as adjuncts to regular drug therapy not as a replacement or substitute.

Follow up was extended for another 3 months, on no supplementation after the trial period of 12 months, to study the effects of withdrawal.

Complete blood profile- including; thiobarbituric acid reactive substances (TBARS) and malondialdehyde (MDA ) were obtained by colorimetric methods. MDA was quantified by a reaction with thiobarbituric acid [20, 21]. Resting electrocardiogram and prothrombin time (on anticoagulants) were done in all the patients at baseline and during follow up, whenever indicated. CoQ levels in the serum were measured by high pressure liquid chromatography based on earlier method [22, 23] at IFTM College of Pharmacy. The technicians measuring the laboratory data were blind to the groups.

Statistical analysis was performed by using Analysis of Variance and Student's t-test for continuous variables. Categorical variables were described as frequencies and percentages, and differences in the two groups were assessed by using Chi square test. A p value of $<0.05$ by two tailed test was considered significant.

\section{RESULTS}

Of the total 67 patients recruited for this trial, 5 were excluded and rest 62 were randomized to intervention $(n=31)$ and placebo group $(n=31)$. Two patients were lost in the follow up and another 2 discontinued the medication due to persistent vomiting. There were 3 deaths in the carniqgel group and 8 in the control during the follow up of 15 months. Causes of heart failure. (n,\%) were; ischemic heart disease $(12,41.3)$ vs $(13,45.4)$, valvular heart disease $(9$, $31.0)$ vs $(10,34.5)$ and cardiomyopathy $(10,34.5)$ vs $(8,27.6)$ in the intervention and control group, respectively. Table $\mathbf{1}$ shows the baseline clinical data in the intervention and control groups. The percentage of men was slightly higher in the control group compared to intervention group. Mean age, height, weight and waist girth revealed no significant differences between the two groups.

Biochemical parameters of oxidative stress; TBARS, and MDA were comparable in the two groups at baseline. However, after 12 months, both the parameters showed significant decline in the carniqgel group without significant reduction in the control group. There was a significant increase in plasma coenzyme Q levels in both the groups, however the increase in CoQ level was several fold greater in the CoQ group compared to the control group (Table 2).

Quality of life visual analogous scale revealed that dyspnea, palpitation and fatigue, which were present at rest, in all the patients, at baseline, showed significantly greater beneficial effects in the intervention group compared to placebo group, after a follow up of 12 months (Table 3). Assessment of physical activity, by distance walked in 6 minutes, revealed that it was comparable at baseline, in the two groups, however, after 12 months of follow up, it was significantly greater in the intervention group than in the control group. Severity of heart failure assessed by NYHA classes, also revealed that the intervention group had greater benefit compared to control the group at 12 months of follow up (Table 3). 
Table 1. Demographic Characteristics of Subjects in the Two Groups. Data is Shown as Mean \pm Standard Deviation

\begin{tabular}{|c|c|c|}
\hline Data & Intervention Group A $(\mathbf{n}=\mathbf{3 1})$ & Control Group B (n=31) \\
\hline Men & $\mathbf{1 2}(\mathbf{4 1 . 3})$ & $\mathbf{1 6 ( 5 5 . 1 )}$ \\
\hline \hline Mean Age & $52.8 \pm 6.4$ & $50.5 \pm 7.7$ \\
\hline Height(Cm) & $160.3 \pm 12$ & $58.4 \pm 6.5^{*}$ \\
\hline Weight(Kg) & $61.5 \pm 7.3$ & $85.8 \pm 9.2$ \\
\hline Waist girth(Cm) & $87.7 \pm 8.7$ & $39.1 \pm 6.7$ \\
\hline Echocardiographic ejection fraction(\%) & $.38 .6+7.6$ & $19.2 \pm 5.6$ \\
\hline Duration of heart failure(months) & $20.0 \pm 4.5$ & \\
\hline
\end{tabular}

$*=\mathrm{P}<0.05$, values were obtained by analysis of varience.

Table 2. Effect of Carniqgel on Oxidative Stress and Plasma Coenzyme Q10 Levels

\begin{tabular}{|c|c|c|c|c|c|c|}
\hline & \multicolumn{3}{|c|}{ Intervention Group } & & Control Group \\
\hline Data & Basal & After 12 Months & After 15 Months & Basal & After 12 Months & After 15 Months \\
\hline \hline TBARS(pmol/l) & $2.2+1.0$ & $1.3 \pm 0.41^{* *}$ & $1.5 \pm 0.43^{*}$ & $2.1 \pm 1.1$ & $2.1 \pm 0.95$ & $2.2 \pm 1.1$ \\
\hline MDA(pmol/l) & $3.0+1.6$ & $1.8 \pm 0.86^{* *}$ & $2.2 \pm 0.78^{*}$ & $3.0 \pm 1.0$ & $2.5 \pm 0.99^{*}$ & $2.6 \pm 1.2$ \\
\hline Serum CoQ(ug/ml) & $0.21+0.11$ & $6.3 \pm 1.7^{* *}$ & $1.5 \pm 0.17^{*}$ & $0.19 \pm 0.10$ & $0.71 \pm 0.15^{*}$ & $0.66 \pm 0.13$ \\
\hline
\end{tabular}

TBARS=Thiobarbituric acid reactive substances,MDA=Malondialdehyde.TNF=Tumor necrosis factor. CoQ=coenzyme Q;P values were obtained by analysis of variance and Chi square test by comparison of intervention and control group and from basal to final and between 12 month and 15 month values $* *=\mathrm{P}<0.01$. $^{*}=\mathrm{P}<0.05$.

Table 3. Effect of Carniqgel on Clinical Manifestations of Heart Failure

\begin{tabular}{|c|c|c|c|c|c|c|}
\hline & \multicolumn{3}{|c|}{ Intervention Group (n=29) } & \multicolumn{3}{c|}{ Control Group (n=29) } \\
\hline Data & Basal & After 12 Months & After 15 Months & Basal & After 12 Months & After 15 Months \\
\hline \hline Decline in symp- Toms(\%). & $96.6 \pm 10.2$ & $55.2 \pm 7.7^{*}$ & $86.6 \pm 7.0^{* *}$ & $98.4 \pm 11.8$ & $80.5 \pm 9.2^{*}$ & $86.3 \pm 10.1$ \\
\hline 6 min walk(Meters) & $208 \pm 15.8$ & $278 \pm 19.8^{* *}$ & $215 \pm 12.8 * *$ & $218.4 \pm 17.6$ & $245.7 \pm 17.1 *$ & $251.2 \pm 17.6^{*}$ \\
\hline Dyspnea(n\%) & $31(100)$ & $13(44.8 \%)^{* *}$ & $21(72.2 \%)^{*}$ & $31(100)$ & $22(75.8 \%)^{*}$ & $22(75.8 \%)$ \\
\hline Fatigue(n\%) & $31(100)$ & $12(41.4 \%)^{* *}$ & $24(82.7 \%)^{*}$ & $31(100)$ & $23(79.2 \%)^{*}$ & $24(82.7 \%)$ \\
\hline NYHA-III-IV(n\%) & $24(100)$ & $11(38.0 \%)^{* *}$ & $22(75.8 \%)^{*}$ & $31(100)$ & $22(75.8 \%)^{*}$ & $22(75.8 \%)$ \\
\hline
\end{tabular}

NYHA=New York Heart Association. $\mathrm{P}$ values were obtained by analysis of varience and Chi square test. By comparison of $12 \mathrm{month}$ with 15 month values $*=\mathrm{P}<0.05, * *=\mathrm{P}<0.02$.

Assessment of symptoms after 15 months showed a worsening of heart failure, decrease in quality of life as well as a decline in physical activity in the carniqgel group, compared to control group, which may be due to cessation of treatment with carniqgel in the intervention group (Table 3 ).

Intention to treat analysis revealed that there was a significant decline in deaths and hospitalizations in the intervention group compared to control group after 12 and 15 months of follow up (Table 4).

At entry to the study, treatment for heart failure including ACE-inhibitors (0.125-10mg/day), beta blockers $(25-100 \mathrm{mg} /$ day), furosemide (20-30mg/day) and digoxin (0.125-0.25mg/ day) was comparable in the two groups. After 12 months of follow up, there was no significant difference among the subjects, receiving ACE-inhibitors, furosemide and dogoxin in the carniqgel group compared to standard drug therapy, in the control group.

\section{DISCUSSION}

This study shows that treatment with a combination of ubiquinol + L-carnitine causes significant improvement in the quality of life assessed by visual analogous scale and physical performance assessed by a 6 min walk test. Our study also showed a significant improvement in NYHA class II-IV heart failure. All the parameters assessing beneficial effects of intervention supplements remained significantly greater during the follow up of one year. However, after 15 months, there was a worsening in these parameters in the intervention 
Table 4. Effect of Carniqgel on Cardiac end Points in the Two Groups

\begin{tabular}{|c|c|c|c|c|c|c|}
\hline & \multicolumn{3}{|c|}{ Intervention Group (n=31) } & \multicolumn{3}{c|}{ Control Group (n=31) } \\
\hline Data & Basal & After 12 Months & After 15 Months & Basal & After 12 Months & After 15 Months \\
\hline \hline Hospitalizations(n\%) & - & $2(6.45 \%)$ & $9(29.0 \%)$ & - & $11(31.45 \%)$ & $12(38.68 \%)$ \\
\hline Total deaths(n\%) & - & $3(9.68 \%)$ & $3(9.68 \%) *$ & - & $8(25.81 \%)$ & $8(25.81 \%)$ \\
\hline Total hospitalizations & - & $5(16.13 \%)$ & $12(38.68 \%)^{* *}$ & - & $19(57.26 \%)$ & $20(64.49 \%)$ \\
\hline And deaths.(n \%) & & & & & & \\
\hline
\end{tabular}

$=\mathbf{P}<\mathbf{0 . 0 5},{ }^{*}=\mathrm{P}<02 ; \mathrm{P}$ value was obtained by comparison of intervention and control group by chi square test, by intention to treat analysis .

group with a decline in serum CoQ levels at 15 months, after cessation of ubiquinol and carnitine at 12 months.

CoQ levels in the blood depend on the metabolic demand in various tissues, and plasma concentrations of $0.6-1.0 \mathrm{ug} / \mathrm{ml}$ are considered as normal. In our study, serum levels of CoQ were lower in both the group (Table 2). Addition of highly bioavailable softsules to treatment regimen was associated with a highly significant 30 fold increase in the serum concentration of CoQ in the carniqgel group after 12 months treatment compared to baseline levels without such an increase in the control group (Table 2). Our results can not be compared with other studies, because we used very highly bioactive formulation. There is a controversy regarding the therapeutic concentration of CoQ in the plasma for patients with heart failure. Langsjoen proposed, a threshold of 2.5 $\mu \mathrm{g} / \mathrm{ml}$, above which marked improvement in heart failure may be observed. In our study, serum levels increased upto $6.3 \pm 1.7 \mathrm{ug} / \mathrm{ml}$ from baseline $0.21 \pm 0.11 \mathrm{ug} / \mathrm{ml}$ in the intervention group, which was associated with marked improvement in heart failure (Table 2). These levels of CoQ are above the postulated threshold as well as two fold greater than the levels reported in a recent study (4) from Italy $(3.25 \pm 1.5 \mu \mathrm{g} / \mathrm{ml})$. This level can not be achieved with low oral doses and commonly available commercial formulations with gelatin or hard gelatin capsules, in the market. Recent studies [16, 17] using such preparations found no beneficial effects of CoQ in heart failure. Oral dosage forms as the formulation factor can influence absorption, bioavailability and bioactivity of the drugs [22, 30]. Soft gelatin capsules representing liquid fills such as carniqgel (Tishcon Corp. Westbury, NY), tend to be better absorbed than hard gelatin capsules and gelatin capsules containing less microfined drugs or a dry powder. It is known that surfactants, as emulsifying agents, at concentrations below their critical micelle concentration might enhance the solubility and dissolution rate of CoQ and carnitine, from dosage forms; thus the drugs become available for absorption.

CoQ treatment in heart failure has been proposed to ameliorate symptoms of heart failure, improve quality of life and exercise capacity and to reduce hospitalization rates and mortality. Most studies have been open level and most of the beta blockers and ACE-inhibitors were not regularly administered.

It is interesting to note that all parameters of CHF showed beneficial effects, also in the control group compared to the baseline levels. These effects appear to be due to administration of ACE inhibitors and beta blockers that are known for their beneficial effects on cardiac function. These cardioprotective effects were significantly greater in the intervention group compared to the placebo group. Longterm follow up on standard drug therapy for several months did not cause greater benefit in the placebo group, indicating that carniqgel appeared to have additional protective effects compared to treatment with standard drug therapy alone. There was a worsening of heart failure and nonsignificant increase in hospitalizations after cessation of carniqgel supplementation in the intervention group, which may be due to a decline in CoQ levels. In an Italian, double blind multicentre study [14], 641 patients with heart failure were randomized to intervention $(\mathrm{n}=322, \mathrm{CoQ} 150 \mathrm{mg} /$ day) and control group $(n=319)$. After one year, 16 patients died in the CoQ group and 21 in the placebo group indicating no difference in mortality. However, there was a significant decline in NYHA class heart failure and hospitalizations due to worsening of heart failure as well as acute left ventricular failure and pulmonary oedema in the CoQ group compared to control group.

In one recent metaanalysis, combined results of 9 trials were weightened according to the sample size in each trial to produce a weighted mean difference [32]. The only three parameters with adequate numbers of subjects for meaningful analysis were CoQ levels (5 trials), ejection fraction at rest ( 7 trials), and mortality ( 5 trials). For CoQ10 levels (279 patients), the weighted mean difference was 1.5 , indicating an increase of $161 \%$. For ejection fraction at rest (384 patients), the weighted mean difference showed a trend in favor of CoQ of $1.9 \%$. For mortality (836 patients), the odds ratio for reduction in the CoQ group was 0.76 (95\% confidence interval 0.43 to 1.37 ). Several of the individual trials reported positive outcomes for some parameters such as symptom class and exercise capacity. It is clear from this metaanalysis that there were inadequate number of patients in the trials for meaningful results. It seems that to detect $2 \%$ reduction in mortality, 2100 patients would be required in each group for meaningful conclusions.

TBARS and MDA which are indicators of oxidative stress, showed a significant greater decline in the intervention group, indicating that scavenging of free radicals may be a possible mechanism of beneficial effects in patients receiving carniqgel. This decline in the markers of oxidative stress associated with heart failure, may be due to repair of CoQ and possibly, carnitine deficiency, which is commonly ob- 
served in these patients [24-26, 31]. Ubiquinol and carnitine are known to provide beneficial effects by their antioxidant and bioenergetic activity. [3-10,31]. Increased availability of CoQ10 may enhance bioactivity of nitric oxide by decreasing superoxide anion and by interacting with speroxide generation and free radical production [24-30, 32]. In presence of oxidative stress, the inactivation rate of nitric oxide to paroxynitrite by superoxide anions may be reduced by CoQ10, which may be a possible explanation for the improvement in exercise capacity in patients with heart failure [32]. Plasma coenzyme $Q$ showed a rise in the control group, although these patients were not supplemented with ubiquinol or coenzyme Q. It is possible, that in CHF, there is increased consumption of antioxidants to fight the free radical stress, which may result into their deficiency. The rise in CoQ status in the control group, may also be due to a decrease in oxidative stress and catecholamines that are higher in heart failure. It is possible that standard drug therapy with ACE inhibitors and beta blockers may spare CoQ and enhance its synthesis, due to a decline in oxidative stress and inflammation [1-3].

There is evidence of an immune activation in CHF [1-4]. Apart from sparing of ATP, CoQ and carnitine may have modest immune activation function [9-14], which are protective against heart failure. CoQ is quite rich, normally in the healthy myocardium. Myocardial biopsies from patients with various cardiovascular diseases [24-26], have demonstrated low concentrations of $\mathrm{CoQ}$, compared with levels in healthy human subjects [26, 27]. Serum CoQ concentrations were lower than normal, in this study, compared to those reported in healthy subjects in other studies [22, 23, 27-30] (Table 2). CoQ deficiency in conjunction with free radical stress, present in heart failure patients, may cause a decline in immune activation, resulting into greater concentration of proinflammatory cytokines and worsening of heart failure. CoQ administration in these patients, can repair the deficit, and improve immune status, resulting in to decreased production of TNF-alpha and IL-6 with no adverse effect on antiinflammatory IL-10. In one study, lower concentrations of CoQ and higher concentration of TNF-alpha in patients with heart failure have been observed [28]. The dosage of CoQ was only $60 \mathrm{mg} /$ day in this study, which is insufficient to produce therapeutic blood levels $(>3.00 \mathrm{mg} / \mathrm{L})$ and reduction in TNF-alpha. The Tishcon study also showed an improvement in NYHA class heart failure as well as in the quality of life, in the presence of a 30 fold increase in CoQ levels and a decline in oxidative stress. It is possible that changes in biochemical and clinical parameters of heart failure may be due to increased CoQ status in these patients. We did not observe any serious adverse effects of carniqgel in this study. Longterm follow up studies $[8,11,20,23,31,32]$ with higher doses of CoQ upto $2700 \mathrm{mg} /$ day and carnitine upto $15 \mathrm{~g} /$ day have shown only mild gastric manifestations, such as nausea, vomiting and loose stools, without any serious adverse effects in heart failure and heart disease.

Peak plasma levels are attained 5-10 hours later and the mean plasma half life is 34 hours, after oral administration of CoQ. Addition of CoQ as a oily matrix or with vitamin E, may cause a rapid absorption and increased bioavailability, compared to in the powder form. CoQ is sequestrated as chylomicrons and deposited largely in the liver, after absorption, as part of very low density lipoproteins $[23,29,30]$. It is a fat soluble compound largely excreted via the biliary tract and more than $50 \%$ of the dose, is eliminated in the feces.

There is complete absorption of orally administered Lcarnitine via an active transport system located within the intestine mucosa of the duodenum and ileum. Once absorbed, up to $25 \%$ of a given load of L-carnitine may be acylated in the intestinal mucosa and both the free and the acylated forms are distributed all over the body. Following ingestion, peak plasma levels of free and total biologically active L-carnitine occur in 3-5 hours. Average normal total carnitine levels of $59 \mathrm{umol} / \mathrm{L}$ and $51 \mathrm{umol} / \mathrm{L}$ have been reported for men and women, respectively. The plasma half life of L-carnitine may range from 2-15 hours in humans. Elimination of carnitine is largely via renal excretion, but it is highly conserved in the kidney and greater than $90 \%$ of the filtered load of carnitine is reabsorbed, in the proximal tubules of the kidneys and is returned to the circulation when plasma level decreases. Among healthy volunteers, oral administration of $2 \mathrm{~g}$ L-carnitine, may produce levels of 69 umol/L. Carnitine levels in the tissues are generally several fold greater than those in the plasma, suggesting that an effective concentrating mechanism exists. Approximately $95 \%$ of the body stores ( $25 \mathrm{~g}$ of L-carnitine) is found in skeletal muscles and heart, with less in liver, kidney and other tissues $(4 \%)$.

In brief, the results of our study provide further evidence that ubiquinol $+\mathrm{L}$-carnitine administration for longterm, may be associated with improvement in NYHA class heart failure, quality of life and exercise capacity, as well as hospitalizations and deaths, with its increased blood level. Higher dosages of CoQ10 (270mg/day) in highly bioavailable solution form, can markedly enhance the plasma concentration of CoQ10 upto $6.3 \mathrm{ug} / \mathrm{ml}$ without any adverse effects for a long period of one year.

\section{ACKNOWLEDGEMENTS}

We are thankful to Tishcon Corporation,Westbury, NY, USA, for providing unrestricted grant to complete this study.

\section{REFERENCES}

[1] Mann DL. Inflammatory mediators and the failing heart: past, present and the foreseeable future. Circ Res 2002; 91: 988-98.

[2] Anker SD, von Haehling S. Inflammatory mediators in chronic heart failure. An overview. Heart 2004; 90: 464-70.

[3] Rauchhaus M, Coats AJ, Anker SD .The endotoxin -lipoprotein hypothesis. Lancet 2000; 356: 930-3.

[4] Belardedinelli R, Mucaz A, Lacalaprice F, et al. Coenzyme Q10 improves contractility of dysfunctional myocardium in chronic heart failure. Biofactors 2005; 25: 137-46.

[5] Silver MA, Langsjoen PH, Szabolcs Szabo, Patil H, Zelinger A. Effect of atorvastatin on left ventricular diastolic function and ability of coenzyme Q10 to reverse that dysfunction. Am J Cardiol 2004; 94: 1306-10.

[6] Singh RB, Pella D, Neki NS, et al. Mechanism of acute myocardial infarction study (MAMIS). Biomed Pharmacother 2004; 58(Suppl): 111-5.

[7] Singh RB, Pella D, Sharma JP, et al. Increased concentrations of lipoprotein(a), circadian rhythms and metabolic reactions, evoked by acute myocardial infarctions, associated with acute reactions, in relation to large breafast. Biomed Pharmacother 2004; 58(Suppl): 116-23.

[8] Singh RB, DeMeester F, Juneja L, Mechirova V, Pella D. New risk factors of heart failure. Eur Heart J 2007; 8: 1038-9. 
[9] Sinatra ST, Sinatra J. L-Carnitine and the Heart. Keats Publishing, NTC Contemporary Publishing Group, Chicago 1999; 7-35.

[10] Hoff-man-Bang C, Swedberg K, Renhnqvist N, Astrom H. Coenzyme Q10 as adjunctive treatment of chronic heart failure. J Cardiac Failure 1995; 1: 101-7.

[11] Bhagvan HN, Chopra RK. Potential role of ubiquinone (coenzyme Q10) in pediatric cardiomyopathy. Clin Nutr 2005; 24: 331-8.

[12] Berman M, Erman A, Ben-Gal T, et al. Coenzyme Q10 in patients with end stage heart failure awaiting cardiac transplantation. A randomized, placebo controlled study. Clin Cardiol 2004; 27: 295-9.

[13] Langsjoen PH, Langsjoen PH, Folkers K. Longterm efficacy and safety of coenzyme Q10 therapy for idiopathic dilated cardiomyopathy. Am J Cardiol 1990; 65: 521-3.

[14] Morisco C, Trimarco B, Condorelli M. Effect of coenzyme Q10 therapy in patients with congestive heart failure, alongterm multicentre study. In: Folkers K, Mortensen SA, Littarru GP, Yamagami T, Lenaz G, Eds, Seventh international symposium on biomedical \& clinical aspects of coenzyme Q; Clin Invest 1993; 71: 134-6.

[15] Mortensen SA, Vadhanavikit S, Baandrup U, Folkers K. Longterm coenzyme Q10 therapy: a major advance in the management of resistant myocardial failure. Drugs Exp Clin Res 1985; 11: 581-93.

[16] Watson PS, Scalia GM, Galbraith A, Burstow DJ, Bett N, Aroney $\mathrm{CN}$. Lack of effect of coenzyme Q on left ventricular function in patients with congestive heart failure. J Am Coll Cardiol 1999; 33: 1549-52.

[17] Khatta M, Alexander BS, Krichten CM, et al. The effect of coenzyme Q in patients with congestive heart failure. Ann Intern Med 2000; 132: 636-40.

[18] Singh RB, Niaz MA, Agarwal P, Beegum R, Rastogi SS, Sachan DS. A randomized, double blind, placebo controlled trial of Lcarnitine in suspected acute myocardial infarction. Postgrad Med 1996; 72: 45-50.

[19] Kumar A, Singh RB, Saxena M, et al. Effect of carni Q-gel (ubiquinol and carnitine) on cytokines in patients with heart failure in the Tishcon study. Acta Cardiol 2007; 62: 349-54.
[20] Esterbaur H, Cheeseman K. Determination of aldehyde peroxidation products MDA and 4 hydroxynonenal. Methods Enzymol 1990; 186: 407-21.

[21] Niato C, Kawamura M, Yamamoto Y. Lipid peroxides as the initiating factor of atherosclerosis. Ann NY Acad Sci 1993; 676: 27-45.

[22] Chopra RK, Goldman R, Sinatra ST, Bhagavan HN. Relative bioavailability of coenzyme $\mathrm{Q}$ formulations in human subjects . Int J Vitam Nutr Res 1998; 68: 109-13.

[23] Edlund PO. Determination of CoQ, alpha-tocopherol and cholesterol in biological samples by couple - column liquid chromatography with colorimetric and ultraviolet detection. J Chromatogr 1988 425: 87-97.

[24] Littarru GP, Ho L, Folkers K. Deficiency of coenzyme Q10 in human heart disease II. Int J Vitam Nutr Res 1972; 42: 291-5.

[25] Folkers K, Littarru GP, Ho L, Runje TM, Havacoda S, Cooley D. Evidence for a deficiency of coenzyme Q10 in human heart disease. Int J Vitam Res 1970; 40: 380-90.

[26] Folkers K, Baker L, Richardson PC, et al. Biomedical and Clinical Aspects of Coenzyme Q. In: Yamamura Y, Folkers K, Ito Y, Eds. Elsevier, Amsterdam 1980; Vol. 2: p. 449.

[27] Mortensen SA. Perspective on therapy of cardiovascular diseases . Clin Invest 1993; 71S: 116-23.

[28] Berman M, Erman A, Ben-Gal T, et al. Coenzyme Q10 in patients with end stage heart failure awaiting cardiac transplantation. A randomized, placebo controlled study. Clin Cardiol 2004; 27: 295-9.

[29] Hughes K, Lee BL, Feng X, Lee J, Ong CN. Coenzyme Q10 and differences in coronary heart disease risk in Asian Indians and Chinese. Free Rad Biol Med 2002; 32: 132-8.

[30] Wahlqvist ML, Wattanapenpaiboon N, Savige GS, Kannar D. Bioavailability of two different formulations of coenzyme Q10 in healthy subjects. Asia Pac J Clin Nutr 1998; 7: 37-40.

[31] Haeckel R, Raiser E, Oellerich M, Siliprandi N. Carnitine metabolism, function and implication. J Clin Chem Clin Metab 1990; 28: 291-5.

[32] Rosenfeldt F, Hilton D, Pepe S, Krum H. Systemic review of effect of coenzyme Q10 in physical exercise, hypertension and heart failure. Biofactors 2003; 18: 91-100.

(c) Mechirova et al.; Licensee Bentham Open.

This is an open access article licensed under the terms of the Creative Commons Attribution Non-Commercial License (http://creativecommons.org/licenses/by$\mathrm{nc} / 3.0 /$ ) which permits unrestricted, non-commercial use, distribution and reproduction in any medium, provided the work is properly cited. 